\section{Imaging of retinal and choroidal vascular tumours}

H Heimann, F Jmor and B Damato

Eye (2013) 27, 208-216; doi:10.1038/eye.2012.251; published online 30 November 2012

Keywords: choroidal haemangioma; vasoproliferative retinal tumour; retinal haemangioblastoma; SD-OCT; EDI-OCT; autofluorescence

\section{Introduction}

Intraocular vascular tumours comprise choroidal haemangiomas, retinal haemangioblastomas, vasoproliferative tumours, and very rare lesions, such as cavernous retinal angiomas and arterio-venous malformations.

The treatment of retinal and choroidal vascular tumours has progressed considerably in recent years. ${ }^{1,2}$ In addition to conventional radiotherapy, cryotherapy, and laser photocoagulation, new therapeutical options include anti-VEGF pharmacotherapy, proton beam radiotherapy, photodynamic therapy, and small gauge vitrectomy. ${ }^{3-8}$ These have been reviewed elsewhere and are therefore not discussed in the present article.

These therapeutic advances are, in part, due to the tremendous advances in ophthalmic imaging that are now routine. Spectral domain optical coherence tomography (SD-OCT), enhanced depth imaging optical coherence tomography (EDI-OCT), wide-angle photography/angiography/autofluorescence imaging all provide qualitative and quantitative information that previously was unavailable. $^{9-13}$ These methods have become indispensable in the management of patients with a vascular tumour. ${ }^{2}$ Because of their novelty, there is scope for an up-to-date discussion of their relevance to vascular tumours of the eye.

In this article, we review the role of novel imaging techniques in the management of the more common vascular tumours of the
Accepted: 28 October 2012

Published online:

30 November 2012

\section{haemangioblastoma.}


eye, that is, choroidal haemangiomas, vasoproliferative tumours, and retinal haemangioblastomas.

\section{Recent developments in imaging of the ocular fundus}

\section{Wide-angle photography and angiography}

With wide-angle photography systems, multiple or extensive areas of interest can be visualized on one photograph. Sequential images can now be reviewed in high resolution side by side on a computer screen, making it easier to detect change.

RetCam (Clarity Medical Systems, Inc., Pleasanton, CA, USA) combines a fiberoptic digital photography system. RetCam is mainly restricted to paediatric ophthalmology. ${ }^{14}$

The Panoret-1000 (Medibell Medical Vision

Technologies, Ltd., Haifa, Israel) combines a contact wide-angle camera with a fiberoptic light source that is placed on the sclera. ${ }^{9,15}$ It produces excellent pictures with high resolution and natural colours, and has successfully been deployed in ocular oncology centres. ${ }^{9,15}$ At the time of writing this article, the Panoret-1000 is no longer commercially available.

Optomap ultra-widefield imaging (Optos PLC, Dunfermline, UK) is a non-contact system incorporating a scanning laser ophthalmoscope with two wavelengths (532 and $633 \mathrm{~nm}$ ). The main advantages are the high resolution, the extensive focal depth, the ease with which sequential images are compared, the image quality in patients with media opacities, and its tolerance by photophobic patients. ${ }^{16}$ It identifies macular pathology as effectively as conventional high-resolution digital imaging. ${ }^{16}$ One disadvantage is that some areas of the retinal periphery can be obscured by eyelashes, vitreous opacities, or the edge of an intraocular lens. ${ }^{16}$ Furthermore, the system generates dual colour images (red and green) so that the apparent colour of some vascular tumours can be very different from the true clinical appearance.

RetCam, Optomap, and cSLO angiography systems all enable wide-angle fluorescein angiography. With wide-angle contact lenses, ICG angiography is also possible.

\section{$S D-O C T$}

Standard OCT has been in clinical use since $1995 .{ }^{17}$ The introduction of the high-resolution SD-OCT represents a major advance. ${ }^{13,17,18}$ More scans with an axial resolution up to $5 \mu \mathrm{m}$ are obtained more rapidly, also covering a larger area of the fundus. ${ }^{13,18}$ Individual retinal layers up to the junction of the photoreceptor inner and outer segments can now be visualized. ${ }^{13}$ With vascular tumours, SD-OCT scans are mainly used for the detection and quantification of macular changes. ${ }^{17}$ Another important application is the detection of retinal changes associated with vascular tumours, for example, epiretinal membranes or retinal atrophy in areas overlying a vascular tumour. SD-OCT has only a minor role in the differential diagnosis of vascular tumours, as this technique is useful only for the examination of posterior lesions and images only the superficial $1 \mathrm{~mm}$ of the retina and choroid. ${ }^{18,19}$

Modern SD-OCT scanners also enable 3D reconstruction of retinal and superficial choroidal tumours. ${ }^{17,20}$ With new montage techniques, an area of $50^{\circ} \times 35^{\circ}$ can be analysed..$^{21}$

\section{EDI-OCT}

With standard OCT, the choroid and sclera show a relatively low resolution due to the light scattering from the retinal pigment epithelium (RPE) and choriocapillaris. ${ }^{18}$ By pushing the OCT objective lens closer to the eye, an inverted image with higher resolution of the choroid can be obtained. ${ }^{22}$ This 'enhanced depth imaging' may be useful in measuring and characterizing lesions $<1 \mathrm{~mm}$ thick that are too thin to be measured with conventional ultrasonography. ${ }^{19}$

\section{Fundus autofluorescence}

Autofluorescence detects the natural emission of light by intraocular structures after excitation by light of a different wavelength. This investigation amplifies and averages multiple sequential images, obtained with modern cSLO machines. ${ }^{12}$ Two different methods of autofluorescence imaging exist: ${ }^{23}$

Fundus autofluorescence is recorded with the $488 \mathrm{~nm}$ excitation and $>500 \mathrm{~nm}$ emission wavelength settings. The main fluorophore is lipofuscin, which is the end-product of phagocytosed photoreceptor outer segments, mostly accumulating within RPE cells. Increased autofluorescence indicates greater metabolic activity of the RPE cells, which occurs before cell death. ${ }^{12}$ An important benefit of autofluorescence lies in detection of disturbances of the RPE-photoreceptor complex, which cannot be identified with other imaging methods. ${ }^{10,12}$ Decreased or absent fluorescence is a sign of dead or permanently damaged RPE cells. ${ }^{12}$

Near-infrared autofluorescence is recorded with the $787 \mathrm{~nm}$ and $>800 \mathrm{~nm}$ settings of indocyanine green angiography. The main fluorophore detected is melanin, which is localized in the apical part of RPE cells. Disturbances in the near-infrared autofluorescence can 
indicate injuries in the RPE cell layer and photoreceptor complex.

Wide-angle autofluorescence is now available using the Optomap system. ${ }^{24}$ Initial results suggest that wide-angle autofluorescence may be more sensitive in identifying peripheral fundus changes than pseudo-colour imaging due to the higher contrast of the images. ${ }^{10}$

\section{Imaging of vascular tumours}

\section{Choroidal haemangioma}

Most circumscribed choroidal haemangiomas can readily be diagnosed with indirect ophthalmoscopy and ultrasonography. ${ }^{1,25}$ They appear as indistinct, nonpigmented, orange-to-red lesions at the posterior pole and near the optic disc. Associated clinical features are exudative retinal detachment, macular oedema, pigmentary changes within the RPE, subretinal fibrosis over the surface of the lesion, and orange pigment.
Circumscribed haemangiomas are often misdiagnosed as choroidal melanoma or metastasis on referral to tertiary centres. $^{25}$

The apparent colour of choroidal haemangiomas varies according to the imaging method used. With scanning laser systems, choroidal haemangiomas may appear brown so that they are more likely to be mistaken for choroidal melanoma (Figure 1). This is of practical importance when evaluating fundus pictures via e-mail. By contrast, the Panoret-1000 system depicts choroidal haemangioma with their true, red colour (Figure 1).

On ultrasonography, choroidal haemangiomas have a high internal acoustic reflectivity matching the reflectivity of the surrounding choroid. This helps to differentiate choroidal haemangiomas from other choroidal tumours, such as melanoma. ${ }^{25}$ Ultrasonography is also used to measure tumour dimensions. Fluorescein angiography adds little diagnostic information. ${ }^{26}$ In typical cases, it demonstrates an early filling of the tumour vessels and choroidal vasculature before filling of the retinal vessels (Figure 2).

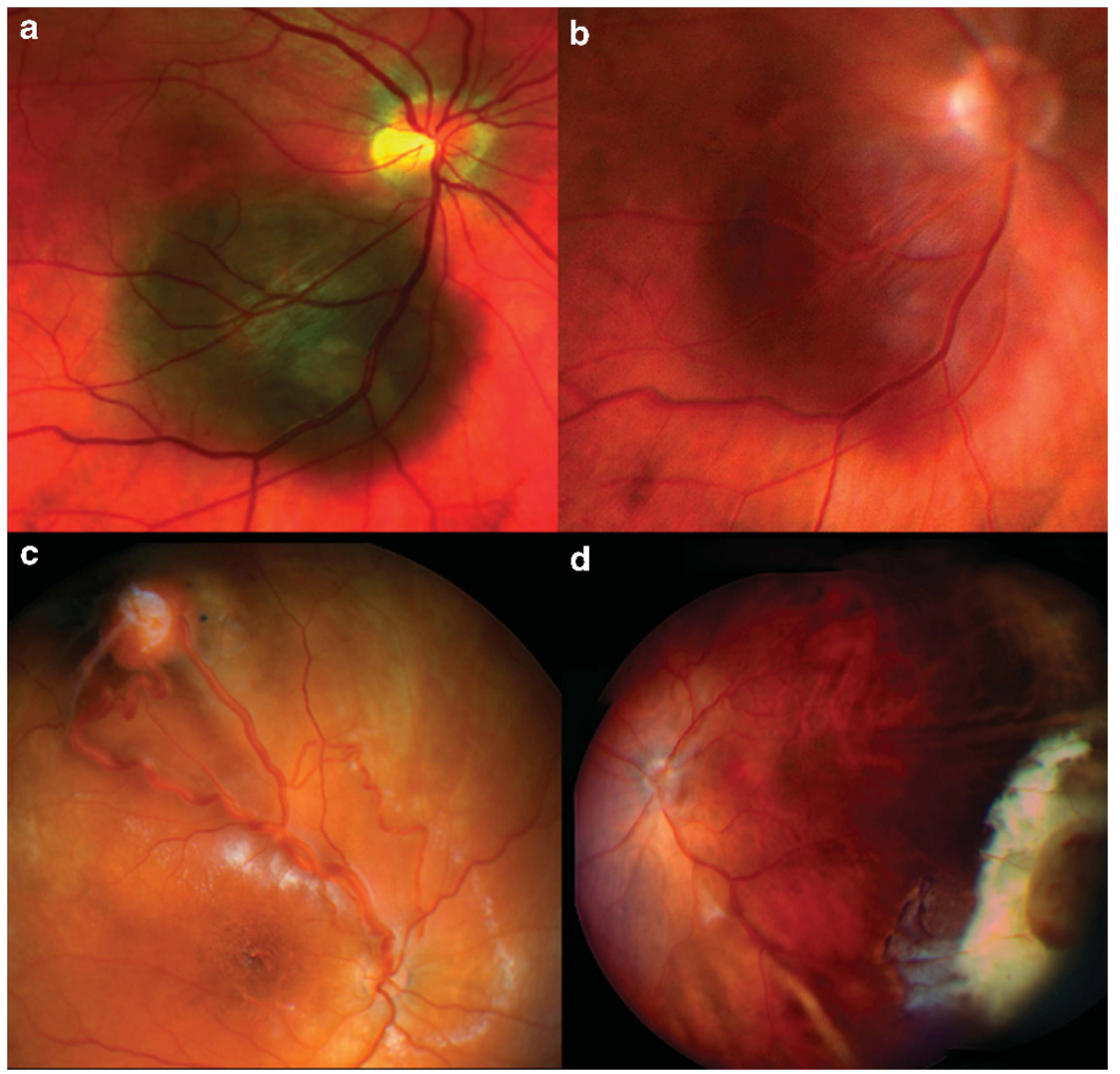

Figure 1 Colour and wide-angle imaging of vascular tumours. (a) Circumscribed haemangioma recorded with the Optomap system. The tumour appears darker and browner and mimics a choroidal melanoma. (b) Same tumour as in (a) imaged with the Panoret camera. A vivid reddish colour of the haemangioma is noted. (c) Peripheral retinal haemangioblastoma imaged with the Panoret camera. The peripheral angioma, dilated feeder vessels, the posterior pole with macular oedema, the optic disc, and the preretinal fibrosis over the tumour can all be documented in one photograph. (d) Vasoproliferative retinal tumour in the temporal periphery imaged with the Panoret system. Typical associated exudative retinal changes and pigmentary changes of the RPE. 
The intrinsic vasculature of haemangiomas displays hyperfluorescence through all phases of the angiography. ${ }^{25}$ ICG angiography and wide-angle ICG angiography are thought to be more useful diagnostically than fluorescein angiography. ${ }^{26}$ The typical ICG

angiographic features of choroidal haemangioma are the appearance of strongly hyperfluorescent intratumoural vessels in the arterial phase, followed by a significant wash-out with hypofluorescence of the tumour relative to the surrounding choiroid in the late phases (30$60 \mathrm{~min}) .{ }^{26}$ Fluorescein and ICG angiography have traditionally been used to monitor the vascular activity of choroidal haemangiomas following treatment. This has now been superseded by monitoring the secondary effects with SD-OCT. ${ }^{8}$

The value of SD-OCT in the assessment of choroidal haemangioma lies in the detection and quantification of associated retinal changes (Figure 3). These include suband intraretinal fluid, retinal schisis, photoreceptor loss, and retinal atrophy. ${ }^{17,20,27,28}$ However, the retina overlying the haemangioma may be normal. ${ }^{20}$ The RPE layer usually shows a dome-shaped uninterrupted elevation over the haemangioma. ${ }^{20,28}$ Disturbances in the
RPE layer corresponding to subretinal fibrosis can also be seen in chronic cases. The macular changes detectable with SD-OCT are foveal cystoid oedema and/or subfoveal fluid. SD-OCT, therefore, is useful for monitoring treatment response, for example, regression of macular oedema or exudative retinal detachment. ${ }^{29}$ Recurrent subretinal fluid following therapy can be detected before the vision deteriorates and before this fluid becomes apparent clinically. ${ }^{29,30}$ At baseline, SDOCT images can also be used to distinguish acute (exudative detachment with preserved retinal architecture) from chronic changes (retinal atrophy). Together with the evaluation of the inner segment/ outer segment photoreceptor line and the integrity of the RPE layer, this information may be useful when considering treatment options and potential outcome (Figure 3).

Preservation of outer retinal thickness and integrity of the inner segment/outer segment photoreceptor line are important prognostic factors for visual recovery following treatment. ${ }^{28}$

Regarding SD-OCT imaging of the haemangioma itself, the anterior surface of the tumour appears

hyporeflective. ${ }^{20}$ This is not detectable using standard

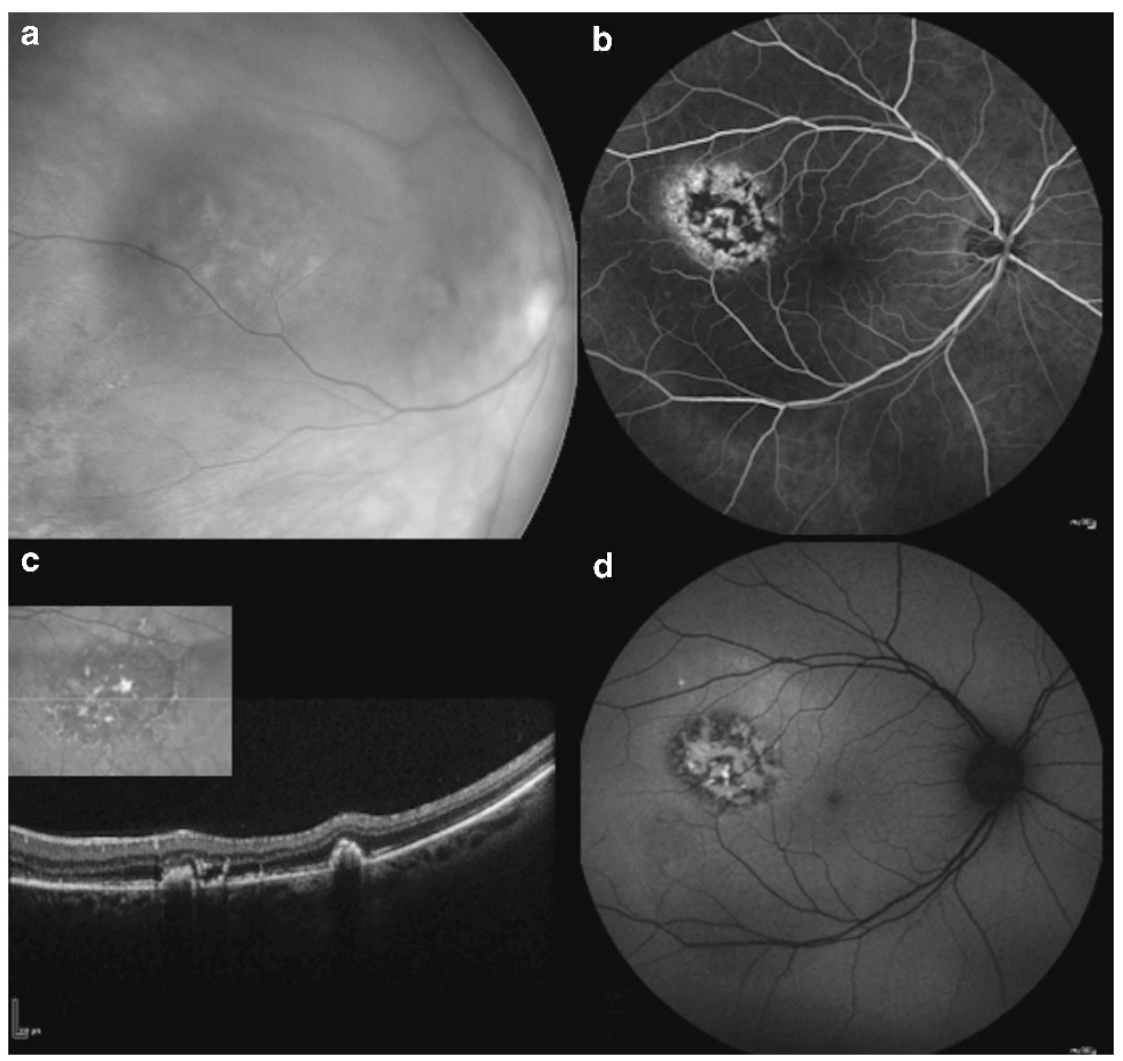

Figure 2 Multimodal imaging of circumscribed choroidal haemangioma. (a) Panoret wide-angle fundus photograph demonstrating the circumscribed choroidal haemangioma. (b) Wide-angle $50^{\circ}$ fluorescein angiography demonstrating hyperfluorescence of the haemangioma in the arterio-venous phase. (c) SD-OCT with fibrotic changes in the RPE layer. The tumour is not visualized with standard SD-OCT settings. (d) Fundus autofluorescence showing hyperfluorescence of the clinically visible orange pigment over the haemangioma. 

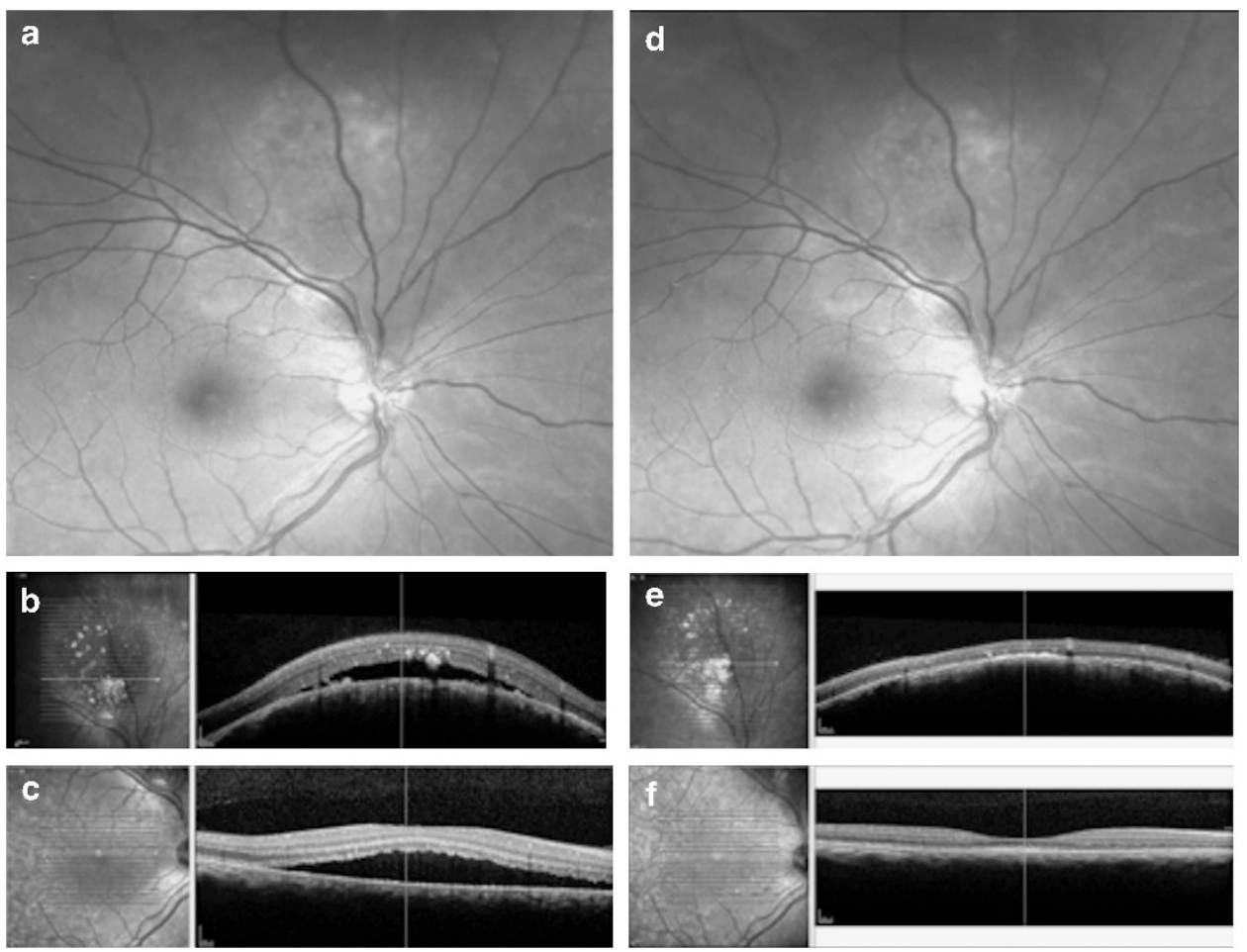

Figure 3 SD-OCT in the management of circumscribed choroidal haemangioma. (a) Pre-treatement Optomap picture of a circumscribed choroidal haemangioma above the disc. (b) SD-OCT over the haemangioma, demonstrating fluid between the photoreceptor and the RPE layer. The RPE layer is interrupted. No details of the haemangioma can be visualized. (c) SD-OCT over the fovea. The exudative detachment extends into the fovea. (d) Same patient 8 months following PDT using standard parameters. On the Optomap picture, no significant differences can be seen. (e) SD-OCT over the tumour following PDT. Regression of subretinal fluid and thinning of the retinal layers over the haemangioma. (f) SD-OCT over the fovea following PDT. Complete regression of the subretinal fluid. No indication for additional treatment.

SD-OCT scans. ${ }^{28}$ Interestingly, details of the superficial choroidal structures reappear under the RPE band following successful treatment of the choroidal haemangioma. ${ }^{28}$ On EDI scans, circumscribed choroidal haemangiomas appear as a low-to-medium reflective band with a homogenous signal and intrinsic spaces. ${ }^{19}$ They can be distinguished from normal surrounding choroidal tissue, but their posterior border is detectable only in tumours with a thickness of $<0.9 \mathrm{~mm}$. ${ }^{19}$

Autofluoresence imaging shows choroidal haemangioma to be hypo-or isofluorescent relative to the surrounding tissue. ${ }^{27}$ The autofluorescence shows mainly a granular pattern. Orange pigment overlying the haemangioma is strongly hyperfluorecent, whereas localized fibrosis and RPE atrophy are hypofluorescent (Figure 2). Fresh subretinal fluid is hyperfluorescent. By contrast, chronic subretinal fluid is hypofluorescent relative to the surrounding tissue. ${ }^{27}$ Following treatment, the relative hypofluorescence of the haemangiomas is more pronounced. ${ }^{27}$

Diffuse choroidal haemangiomas are usually associated with Sturge-Weber syndrome. They more closely resemble a diffuse choroidal angiomatosis than a localized vascular tumour. ${ }^{8}$ On ultrasonography, the extension of the lesion usually exceeds the apparent diameter based on indirect ophthalmoscopy. Wide-angle ICG angiography is useful in determining the extent of the haemangioma. With SD-OCT, associated retinal changes resemble those of occurring in circumscribed haemangioma. ${ }^{31}$ Similarly, the autofluoresence pattern of diffuse choroidal haemangioma is similar to that of circumscribed lesions. ${ }^{27}$

\section{Retinal vasoproliferative tumour}

Retinal vasoproliferative tumours comprise a spectrum of focal-to-diffuse vascularized retinal lesions having a prominent glial component (Figure 1). ${ }^{1,32}$ These usually appear as pink, retinal tumours or diffuse fibrotic thickening, usually in the inferior fundus periphery, often with a haemorrhagic component. Associated changes are exudative retinal detachment, macular oedema, sub-, intra-, and epiretinal fibrosis, hyper- and hypo-pigmentation of the RPE, and cellular infiltrates in the vitreous. Macular changes exist in 59\% of idiopathic vasoproliferative tumours of the retina and in $92 \%$ of 
cases that are associated with other ophthalmlogical diseases. $^{33}$

Because of their pre-equatorial location, many retinal vasoproliferative tumours cannot be photographed with conventional imaging methods, and some are also beyond the reach of wide-angle cameras. Nevertheless, wide-angle photography is useful for monitoring of associated retinal changes. Some vasoproliferative retinal tumours are initially managed by observation, and in such cases, wide-angle photography aids evaluation of the lesions so as to determine the need for treatment. Fluorescein angiography can be helpful in the initial diagnosis of vasoproliferative retinal tumours. For example, it helps to differentiate these from peripheral exudative haemorrhagic chorioretinopathy. ${ }^{34}$ In contrast to retinal capillary haemangioblastoma, retinal feeder vessels are only mildly dilated on fluorescein angiography. Wide-angle angiography is particularly useful in demonstrating the vasoproliferative nature and vascular supply of these lesions. Because of the retinal localization of these lesions, ICG angiography does not add useful information.

Most vasoproliferative tumours of the retina are too peripheral to be imaged with standard SD-OCT. Posterior lesions demonstrate retinal inner layer disorganization and posterior shadowing. ${ }^{35}$ SD-OCT is a useful imaging tool in the management of such lesions. ${ }^{17,33}$ In a large number of cases, the long-term visual disability that is caused by retinal vasoproliferative tumours will be related to retinal changes in the macular region and not by the tumour itself. In particular, there is a high incidence of epiretinal membrane formation and macular oedema. Our current treatment strategy is based on SD-OCT imaging and involves small-gauge vitrectomy with peeling of epiretinal membranes and/or anti-VEGF or steroid injections for macular oedema. This is in addition to treatment of the peripheral vasoproliferative tumour by laser photocoagulation, photodynamic therapy, or brachytherapy.

After initial treatment, SD-OCT is used to monitor treatment response and indicate the need for additional therapy. Following successful ablation of the vasoproliferative tumour, regression of the associated retinal exudative changes occurs. SD-OCT and wideangle autofluroescence imaging demonstrate the extent of any permanent damage in the photoreceptor-RPE complex. Typical findings with SD-OCT are retinal and RPE atrophy. With autofluorescence, hyperfluorescence of areas bordering the exudative changes as well as a relative hypofluorescence in the area affected by the exudative detachment can be seen. Following treatment and regression, the areas of significant retinal damage and atrophy appear hypofluorescent.

\section{Retinal haemangioblastoma}

Retinal haemangioblastomas can affect any part of the fundus and vary greatly in their clinical appearance. ${ }^{36,37}$ They may occur sporadically or with the von Hippel-Lindau syndrome (Figure 1). ${ }^{38,39}$ Small tumours appear as tiny, red dots or as retinal teleangiectasia. On ophthalmoscopy, the angiomatous nature may sometimes be obscured by epiretinal fibrosis so that the only visible clues may be the associated changes in the surrounding retinal vessels. Larger angiomas can appear as a pink-orange mass of coiled vascular structures rather than a large, blood-filled angioma. Dilated feeder vessels and an epiretinal fibrotic reaction are typical of larger haemangioblastomas (Figure 1). Visual loss is caused by intra- and subretinal exudates, macular oedema, exudative retinal detachment, epiretinal membrane formation, and tractional retinal detachment. It is important to identify retinal haemangioblastomas and their associated complications at an early stage as advanced lesions are not usually controlled with conventional laser- or cryotherapy alone. ${ }^{7}$ These large lesions are usually accompanied by severe exudative and vitreoretinal traction. Such advanced disease often results in a blind and painful eye, necessitating enucleation in about $10 \%$ of patients. ${ }^{36}$

Juxtapapillary retinal haemangioblastomas differ clinically from peripheral lesions. ${ }^{39}$ They are classified as endophytic (ie, intravitreal), exophytic (ie, subretinal), or sessile (ie, intraetinal). Small angiomas on the disc initially resemble splinter haemorrhages. When they enlarge, they become visible more as a diffuse thickening of the retina. Only in the advanced stages do they develop a typical angiomatous appearance. These tumours are associated with exudative and glial retinal changes as outlined above. In contrast to peripheral haemangioblastomas, dilated feeder vessels do not develop.

Wide-angle photography is extremely useful in the management of patients with retinal haemangioblastoma, particularly in the presence of the von Hippel-Lindau syndrome (Figures 1 and 4). This is because such patients with VHL can have multiple tumours, with new lesions developing over time. In addition, they often develop a variety of sightthreatening complications that need to be detected and monitored. These include exudation, macular oedema, exudative and tractional retinal detachment, and epiretinal membrane formation (Figures 1 and 4). The panoramic photographic documentation now enables the treating ophthalmologist to analyse changes over time with greater ease and accuracy than was hitherto possible. 

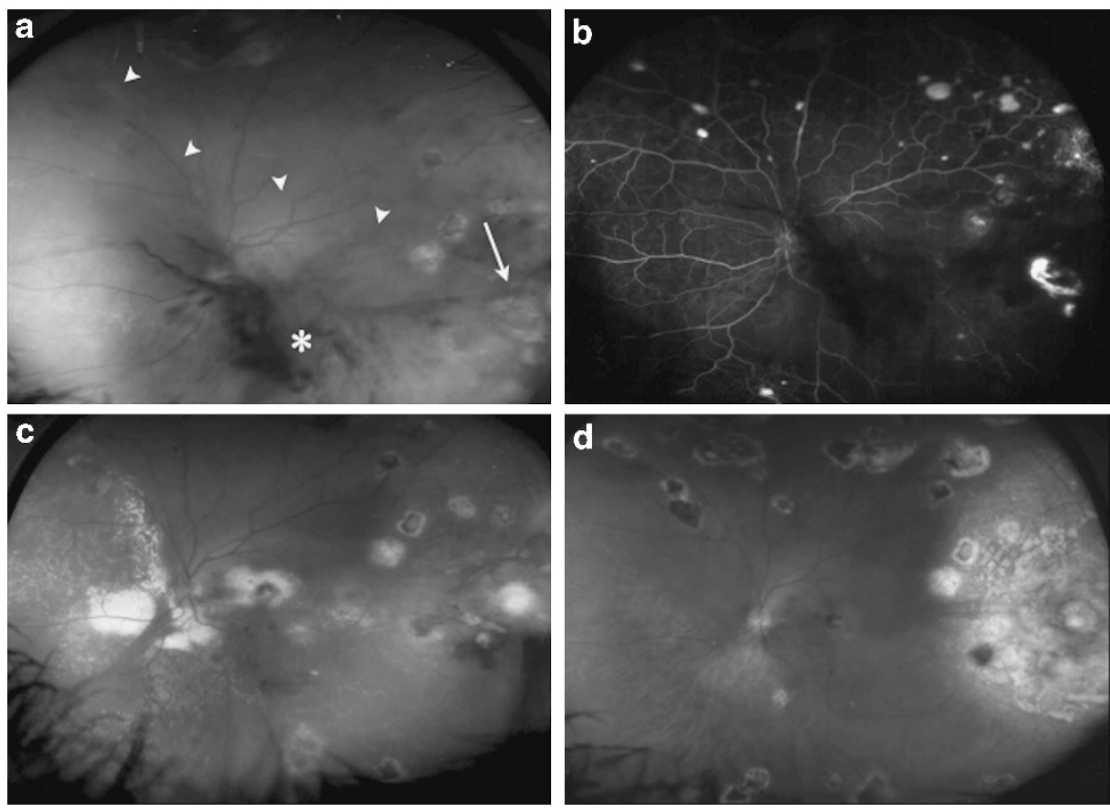

Figure 4 Patient with multiple retinal haemangioblastoma associated with von Hippel-Lindau syndrome in the left eye. The patient was referred following unsuccessful laser and cryotherapy. (a) At the initial examination in our institution, there was a large and active haemangioblastoma in the temporal periphery (arrow) associated with a vitreous haemorrhage $\left(^{*}\right)$ and a near-total exudative retinal detachment (arrowheads). (b) The wide-angle fluorescein angiography highlights more than 20 active haemangioblastoma (visible as leaking spots during the angiography). (c) Same patient 4 months after intitial treatment with brachytherapy and laser. Regression of the large angioma in the temporal periphery. Collection of lipoid exudates in the area of the previous exudative retinal detachment. (d) Same patient 17 months following brachytherapy and multiple sessions of laser photocoagulation. Complete regression of all previously active haemangioblastoma. Regression of the exudative retinal detachment and lipoid exudates.

Fluorescein angiography is still valuable in assessing patients with retinal haemangioblastomas. In addition to feeder vessels, these tumours show early hyperfluorescence during the arterial phase with increasing but variable leakage during the later phases of the angiogram. ${ }^{35}$ Wide-angle angiography greatly facilitates the detection of small angiomas and is more sensitive than indirect ophthalmoscopy and fundus photography (Figure 4). ${ }^{37}$ ICG angiography can be useful in selected cases, particularly with juxtapapillary haemangioblastomas, demonstrating vasculature that would otherwise be obscured by early fluorescein leakage.

The value of SD-OCT in the management of patients with retinal haemangioblastoma lies in the identification and monitoring of associated retinal changes. ${ }^{40}$ These include macular oedema, retinal atrophy, retinal detachment and epi- or subretinal membrane formation, which are not as well demonstrated by indirect ophthalmoscopy and other imaging methods. Early identification and treatment of these effects may have a greater influence on visual outcome than treatment of the tumour itself. When located near the posterior pole, the haemangioblastoma can be visualized on the SD-OCT scan (Figure 5). It appears as an optically-dense, inner-retinal mass with posterior shadowing or thickening and disorganization of the retinal layers. ${ }^{17,40,41}$

Fundus autofluorescence can provide additional information regarding the activity state and viability of the photoreceptor-RPE complex. Areas near exudative retinal changes usually show hyperfluorescence, whereas atrophic or permanently damaged retina appears hypofluorescent.

\section{Summary and conclusion}

The past decade has seen much progress in the management of patients with a vascular tumour of the eye. This advance is closely linked to the development of new imaging methods, in particular, SD-OCT and wideangle photography. When patients are initially managed by observation, SD-OCT and autofluorescence imaging indicate the chances of functional improvement with treatment. Furthermore, early detection of subclinical macular oedema or epiretinal membranes can allow treatment before visual loss occurs, enhancing long-term outcomes.

Thanks to improved imaging, current treatment strategies for vascular tumours have fundamentally changed. Previously, only the tumour was treated, with secondary effects being managed at a later stage; today, 

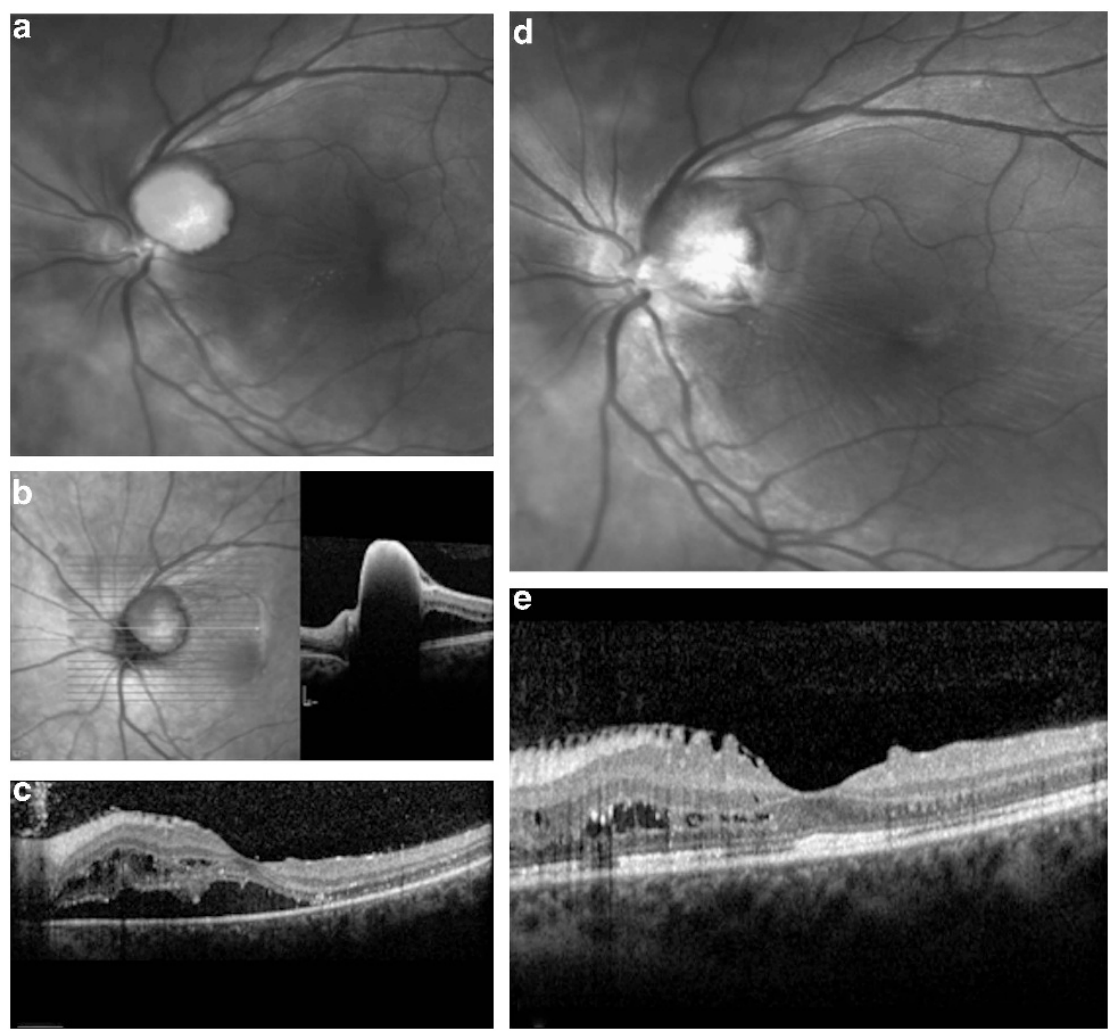

Figure 5 Retinal haemangiblastoma on the disc not associated with von Hippel-Lindau syndrome. (a) Baseline fundus picture showing an endophytic peripapillary angioma with retinal exudates. (b) SD-OCT over the angioma. No retinal structures can be identified over the tumour. The angioma appears as an optically dense inner retinal mass with posterior shadowing. (c) SD-OCT over the macula. Intraretinal exudative changes and exudative retinal detachment involving the fovea. Visual acuity 6/18. (d, e) One year after PDT. (d) Fundus picture demonstrating a fibrotic reaction in the angioma following PDT. Visual acuity 6/9. (e) SD-OCT over the macula. Regression of intraretinal fluid and subretinal fluid following treatment.

both the primary tumour and the secondary effects are treated simultaneously from the outset.

Panoramic fundus photography has become indispensable, with a single image demonstrating not only the activity of the vascular lesion but also the associated exudative retinal changes, epiretinal membranes, areas of retinal traction, and any new lesions. Moreover, sequential high-resolution images greatly facilitate the assessment of disease activity and response to treatment.

New imaging methods are being deployed in routine clinical practice, and these include fundus autofluorescence, wide-angle autofluorescence, nearinfrared autofluorescence, and EDI-OCT. It is likely that these new methods will further improve our understanding of vascular tumours of the retina and choroid and their secondary effects on the eye thereby enhancing the targeted treatment of these rare tumours.

\section{Conflict of interest}

The authors declare no conflict of interest.

\section{References}

1 Heimann H, Damato B. Congenital vascular malformations of the retina and choroid. Eye 2010; 24(3): 459-467.

2 Knutsson KA, De Benedetto U, Querques G, Del Turco C, Bandello F, Lattanzio R. Primitive retinal vascular abnormalities: tumors and telangiectasias. Ophthalmologica 2012; 228(2): 67-77.

3 Shoeibi N, Ahmadieh H, Abrishami M, Poorzand H. Rapid and sustained resolution of serous retinal detachment in Sturge-Weber syndrome after single injection of intravitreal bevacizumab. Ocul Immunol Inflamm 2011; 19(5): 358-360.

4 Zografos L, Egger E, Bercher L, Chamot L, Munkel G. Proton beam irradiation of choroidal hemangiomas. Am J Ophthalmol 1998; 126(2): 261-268.

5 Ziemssen F, Heimann H. Evaluation of verteporfin pharmakokinetics-redefining the need of photosensitizers in ophthalmology. Expert Opin Drug Metab Toxicol 2012; 8(8): 1023-1041.

6 Chan RVP, Yonekawa Y, Lane AM, Skondra D, Munzenrider JE, Collier JM et al. Proton beam irradiation using a lightfield technique for the treatment of choroidal hemangiomas. Ophthalmologica 2010; 224(4): 209-216.

7 Gaudric A, Krivosic V, Duguid G, Massin P, Giraud S, Richard S. Vitreoretinal surgery for severe retinal capillary 
hemangiomas in von hippel-lindau disease. Ophthalmology 2011; 118(1): 142-149.

8 Blasi MA, Tiberti AC, Scupola A, Balestrazzi A, Colangelo $\mathrm{E}$, Valente $\mathrm{P}$ et al. Photodynamic therapy with verteporfin for symptomatic circumscribed choroidal hemangioma: five-year outcomes. Ophthalmology 2010; 117(8): 1630-1637.

9 Shields CL, Materin M, Shields JA. Panoramic imaging of the ocular fundus. Arch Ophthalmol 2003; 121(11): 1603-1607.

10 Heussen FM, Tan CS, Sadda SR. Prevalence of peripheral abnormalities on ultra-widefield greenlight $(532 \mathrm{~nm})$ autofluorescence imaging at a tertiary care center. Invest Ophthalmol Vis Sci 2012; 53(10): 6526-6531.

11 Wong IY, Koizumi H, Lai WW. Enhanced depth imaging optical coherence tomography. Ophthalmic Surg Lasers Imaging 2011; 42(Suppl): S75-S84.

12 Schmitz-Valckenberg S, Holz FG, Bird AC, Spaide RF. Fundus autofluorescence imaging: review and perspectives. Retina 2008; 28(3): 385-409.

13 Wolf S, Wolf-Schnurrbusch U. Spectral-domain optical coherence tomography use in macular diseases: a review. Ophthalmologica 2010; 224(6): 333-340.

14 Salcone EM, Johnston S, VanderVeen D. Review of the use of digital imaging in retinopathy of prematurity screening. Semin Ophthalmol 2010; 25(5-6): 214-217.

15 Pe'er J, Sancho C, Cantu J, Eilam S, Barzel I, Shulman $\mathrm{M}$ et al. Measurement of choroidal melanoma basal diameter by wide-angle digital fundus camera: a comparison with ultrasound measurement. Ophthalmologica 2006; 220(3): 194-197.

16 Csutak a, Lengyel I, Jonasson F, Leung I, Geirsdottir A, Xing $\mathrm{W}$ et al. Agreement between image grading of conventional $\left(45^{\circ}\right)$ and ultra wide-angle $\left(200^{\circ}\right)$ digital images in the macula in the Reykjavik eye study. Eye 2010; 24(10): 1568-1575.

17 Say EAT, Shah SU, Ferenczy S, Shields CL. Optical coherence tomography of retinal and choroidal tumors. Journal of ophthalmology 2012; 2012: 385058.

18 Regatieri CV, Branchini L, Fujimoto JG, Duker JS. Choroidal imaging using spectral-domain optical coherence tomography. Retina 2012; 32(5): 865-876.

19 Torres VLL, Brugnoni N, Kaiser PK, Singh AD. Optical coherence tomography enhanced depth imaging of choroidal tumors. Am J Ophthalmol 2011; 151(4): 586-593.

20 Sayanagi K, Pelayes DE, Kaiser PK, Singh AD. 3D Spectral domain optical coherence tomography findings in choroidal tumors. Eur J Ophthalmol 2011; 21(3): 271-275.

21 Gregori NZ, Lam BL, Gregori G, Ranganathan S, Stone EM, Morante A et al. Wide-field spectral-domain optical coherence tomography in patients and carriers of X-linked Retinoschisis. Ophthalmology 2012; e-pub ahead of print 23 September 2012; doi:10.1016/j.ophtha.2012.07.051

22 Spaide RF. Enhanced depth imaging optical coherence tomography of retinal pigment epithelial detachment in age-related macular degeneration. Am J Ophthalmol 2009; 147(4): 644-652.

23 Kellner U, Kellner S, Weinitz S. Fundus autofluorescence (488 NM) and near-infrared autofluorescence (787 NM) visualize different retinal pigment epithelium alterations in patients with age-related macular degeneration. Retina 2010; 30(1): 6-15.

24 Slotnick S, Sherman J. Panoramic autofluorescence: highlighting retinal pathology. Optom Vis Sci 2012; 89(5): 575-584.

25 Shields CL, Honavar SG, Shields JA, Cater J, Demirci H. Circumscribed choroidal hemangioma: clinical manifestations and factors predictive of visual outcome in 200 consecutive cases. Ophthalmology 2001; 108(12): 2237-2248.

26 Schalenbourg A, Piguet B, Zografos L. Indocyanine green angiographic findings in choroidal hemangiomas: A study of 75 cases. Ophthalmologica 2000; 214(4): 246-252.

27 Ramasubramanian A, Shields CL, Harmon S a, Shields J a. Autofluorescence of choroidal hemangioma in 34 consecutive eyes. Retina 2010; 30(1): 16-22.

28 Liu W, Zhang Y, Xu G, Qian J, Jiang C, Li L. Optical coherence tomography for evaluation of photodynamic therapy in symptomatic circumscribed choroidal hemangioma. Retina 2011; 31(2): 336-343.

29 Kwon HJ, Kim M, Lee CS, Lee SC. Treatment of serous macular detachment associated with circumscribed choroidal hemangioma. Am J Ophthalmol 2012; 154(1): 137-145.

30 Zhang Y, Liu W, Fang Y, Qian J, Xu G, Wang W et al. Photodynamic therapy for symptomatic circumscribed macular choroidal hemangioma in Chinese patients. Am J Ophthalmol 2010; 150(5): 710-715.

31 Tsipursky MS, Golchet PR, Jampol LM. Photodynamic therapy of choroidal hemangioma in sturge-weber syndrome, with a review of treatments for diffuse and circumscribed choroidal hemangiomas. Surv Ophthalmol 2011; 56(1): 68-85.

32 Hiscott $\mathrm{P}, \mathrm{Mudhar} \mathrm{H}$. Is vasoproliferative tumour (reactive retinal glioangiosis) part of the spectrum of proliferative vitreoretinopathy? Eye 2009; 23(9): 1851-1858

33 Cohen VML, Shields CL, Demirci H, Shields Ja. Iodine I 125 plaque radiotherapy for vasoproliferative tumors of the retina in 30 eyes. Arch Ophthalmol 2008; 126(9): 1245-1251.

34 Tsui I, Jain A, Shah S, Schwartz SD, McCannel Ta. Ultra widefield imaging of peripheral exudative hemorrhagic chorioretinopathy. Semin Ophthalmol 2009; 24(1): 25-28.

35 Turell ME, Singh AD. Vascular tumors of the retina and choroid: diagnosis and treatment. Middle East African J Ophthalmol 2010; 17(3): 191-200.

36 Wong WT, Agrón E, Coleman HR, Tran T, Reed GF, Csaky K et al. Clinical characterization of retinal capillary hemangioblastomas in a large population of patients with von Hippel-Lindau disease. Ophthalmology 2008; 115(1): 181-188.

37 Kreusel K-M, Bechrakis NE, Krause L, Neumann HPH, Foerster MH. Retinal angiomatosis in von Hippel-Lindau disease: a longitudinal ophthalmologic study. Ophthalmology 2006; 113(8): 1418-1424.

38 Kreusel KM, Bechrakis NE, Heinichen T, Neumann L, Neumann HP, Foerster MH. Retinal angiomatosis and von Hippel-Lindau disease. Graefe's Arch Clin Exp Ophthalmol 2000; 238(11): 916-921.

39 Kreusel K-M, Bechrakis NE, Neumann HPH, Schmidt D, Foerster MH. Solitary juxtapapillary capillary retinal angioma and von Hippel-Lindau disease. Can J Ophthalmol 2007; 42(2): 251-255.

40 Shields CL, Materin M a, Shields Ja. Review of optical coherence tomography for intraocular tumors. Curr Opin Ophthalmol 2005; 16(3): 141-154.

41 Shields CL, Mashayekhi A, Luo CK, Materin M a, Shields Ja. Optical coherence tomography in children: analysis of 44 eyes with intraocular tumors and simulating conditions. J Pediatr Ophthalmol Strabismus 2004; 41(6): 338-344. 